\title{
Upregulation of Endogenous HMOX1 Expression by a Computer-Designed Artificial Transcription Factor
}

\author{
Hongfeng Guo, ${ }^{1}$ Yi Tian, ${ }^{2}$ Hai Lu, ${ }^{1}$ Yong Wei, ${ }^{1}$ and Dajun Ying ${ }^{1}$ \\ ${ }^{1}$ Department of Anatomy, Third Military Medical University, Gao-Tan-Yan Street, Sha-Ping-Ba District, Chongqing 400038, China \\ ${ }^{2}$ Department of Immunology, Third Military Medical University, Gao-Tan-Yan Street, Sha-Ping-Ba District, \\ Chongqing 400038, China
}

Correspondence should be addressed to Dajun Ying, ydj96@yahoo.com.cn

Received 1 March 2010; Revised 25 May 2010; Accepted 17 June 2010

Academic Editor: Kostas Iatrou

Copyright (c) 2010 Hongfeng Guo et al. This is an open access article distributed under the Creative Commons Attribution License, which permits unrestricted use, distribution, and reproduction in any medium, provided the original work is properly cited.

\begin{abstract}
Heme oxygenase-1 (HO-1) is well known as a cytoprotective factor. Research has revealed that it is a promising therapeutic target for cardiovascular diseases. In the current study, an HMOX1 (HO-1 gene) enhancer-specific artificial zinc-finger protein (AZP) was designed using bioinformatical methods. Then, an artificial transcription factor (ATF) was constructed based on the AZP. In the ATF, the p65 functional domain was used as the effector domain (ED), and a nuclear localization sequence (NLS) was also included. We next analyzed the affinity of the ATF to the HMOX1 enhancer and the effect of the ATF on endogenous HMOX1 expression. The results suggest that the ATF could effectively upregulate endogenous HMOX1 expression in ECV304 cells. With further research, the ATF could be developed as a potential drug for cardiovascular diseases.
\end{abstract}

\section{Introduction}

Heme oxygenase-1 (HO-1) catalyzes the rate-limiting reaction in heme degradation (the oxidative cleavage of heme to yield carbon monoxide, free iron, and biliverdin). Besides directly eliminating heme, $\mathrm{HO}-1$ plays a cytoprotective role via its catalytic products with antioxidant, antiinflammatory, antiapoptotic, and cell-signaling properties [1]. Research has revealed that HMOX1 is a cardiovascular system protective gene, which could be adopted as a therapeutic target [2-8].

Artificial transcription factor (ATF) technology is based on the resemblance to natural transcription factors [9]. An ATF consists of a DNA-binding domain (DBD) and an effector domain (ED). It is well known that an ED retains its function when fused to other DBDs [10]. Thus, to construct a desired ATF, the key work is to construct an appropriate DBD. The $\mathrm{Cys}_{2}-\mathrm{His}_{2}$ zinc finger protein, which is composed of several zinc finger motifs, is ubiquitous among natural transcription factors and the most widely used DBD in ATF technology [11]. A single zinc finger motif consists of approximately 30 amino acids, with a simple $\beta \beta \alpha$ fold stabilized by hydrophobic interactions and chelation of a zinc ion. Presentation of the $\alpha$-helix into the major groove of DNA allows for recognition to a base-pair triplet. Variation of the specificity-related amino acid residues in the $\alpha$-helix always changes target specificity of the zinc finger motif.

Phage display technology has played a major role in selecting artificial zinc-finger proteins (AZPs) with highest specificity and affinity in the past decade [12]. However, the tedious and time-consuming procedure is a problem. The one-to-one relationship between base-pair triplets and specificity-related amino acid residues could be summarized as a "recognition code table," which suggests the possibility for the AZP rational design [13]. With the rapid development of bioinformatics, researchers have paid more and more attention to this novel method. In the current study, we designed an HMOX1 enhancer-specific AZP by ZF tools 3.0 [14], which is an online server based on the "recognition code table". Then, we constructed an ATF based on the AZP and confirmed that it could upregulate endogenous HMOX1 expression in ECV304 cells. We demonstrated that the application of bioinformatics in ATF technology provides a facile way to upregulate HMOX1 expression. This method could probably be applied to other disease-related genes as well. 


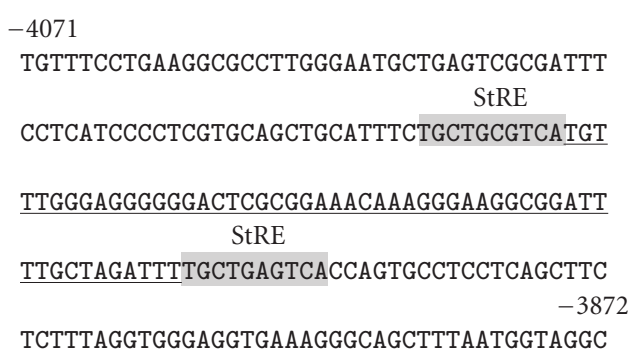

Figure 1: The DNA sequence selected for prescreening. A $54 \mathrm{bp}$ DNA sequence between the two stress response elements (StREs) of human HMOX1 enhancer (underlined) was selected for prescreening.

\section{Materials and Methods}

2.1. ZF Tools 3.0 Designing. ZF tools 3.0 (http://www.scripps .edu/mb/barbas/zfdesign/zfdesignhome.php) is a website which provides several tools for selecting zinc finger protein (ZFP) target sites and for designing the proteins that will target them [14]. In the current study, the first two tools ("Search DNA Sequence for Contiguous or Separated Target Sites" and "Design a Zinc Finger Protein") were explored.

The sequence between the two stress response elements (StREs) of human HMOX1 enhancer was submitted to ZF tools 3.0 to search AZP target sites (Figure 1). Parameter settings are (1) "Triplets to search" included all available triplets (GNG, GN(ACT), ANN, CNN, and TNN). (2) "ZF set" was "total" and "Contiguous targets" radio button was selected. (3) "Minimum target size" was "18". After that, several candidate target sites were found.

Due to the large number of target sites that ZF Tools 3.0 may identify, four scoring functions ("Base", "XNN", "GNN", and "GACT") were implemented to assist users in selecting promising target sites. As a rule in ZF Tools 3.0, the higher the score, the better. However, different scoring functions may generate conflicting results. In the current study, the "Base" function, which uses data from ELISA specificity graphs in order to predict the total number of off-target sites that a given ZFP may recognize, was used since it was the most sophisticated function. In addition, the target site overlap (TSO) interaction may decrease the independent modularity of the triplets and thereby should be avoided. According to these principles, the sequence with the top score and without TSO was selected as the best target site for the AZP, which was then submitted to $\mathrm{ZF}$ tools 3.0 again to obtain the amino acid sequence of the corresponding AZP.

2.2. SWISS-MODEL Assessing. SWISS-MODEL (http://swissmodel.expasy.org/) is a fully automated protein structure homology-modeling server which provides multiple tools for protein modeling [15-17]. In the current study, the amino acid sequence of the AZP was submitted to "Sequence Feature Scan" (http://swissmodel.expasy.org/ space/index.php?func=tools_sequencescan1) for secondary structure prediction, prediction of disordered regions, and assignment of domains in the target sequence. The sequence was then submitted to "Automated Modeling Mode" (http://swissmodel.expasy.org/workspace/index.php? func=modelling_simple1) for homology modeling. After that, "Anolea" [18] and "Verify3D" [19] options were selected for assessing the packing quality and the structure of the AZP.

2.3. ATF Vector Construction. The DNA coding sequence of the AZP was optimized and synthesized by Shanghai Sangon. Then, it was cloned into the PUC57 vector to obtain PUC57-AZP. The pcDNA3.1-NLS-eGFP-p65-flag vector, which contains the coding sequences of the functional domain of p65 (which was used for the effector domain in the current study) and the nuclear localization sequence (NLS) (CCCAAGAAGAAGAGGAAGGTG), was a kind gift from Dr. Philip Gregory (Sangamo BioSciences). PUC57-AZP and pcDNA3.1-NLS-eGFP-p65-flag were double digested by restriction endonucleases KpnI (AflII) and BamHI to obtain the AZP and NLS-p65-flag (p65-flag) fragments. The two fragments were then recombined to construct pcDNA3.1NLS-AZP-p65-flag (pcDNA3.1-AZP-p65-flag), which was regarded as the ATF vector (Figure 2). The two ATF vectors with or without NLS were subsequently verified by DNA sequencing.

2.4. Cell Culture and Transfection. In the current study, ECV304 cell line was used for intracellular tests. ECV304 cell line was regarded as a human endothelial cell line before. However, recent DNA profiling and cytogenetic studies showed that it was a derivative of the T24 bladder carcinoma [20]. ECV304 cells were cultured in DMEM (high glucose) containing $10 \%$ FBS and $\mathrm{ZnCl}_{2}(0.1 \mathrm{mM})$ under a humidified atmosphere of $5 \% \mathrm{CO}_{2}$ and $95 \%$ air at $37^{\circ} \mathrm{C}$. For transfection, cells were cultured in plates 1 day before and transfected using the Lipofectamine 2000 (Invitrogen, Carlsbad, CA, USA) according to the manufacturer's instructions. PUC57-AZP (regarded as the AZP vector), pcDNA3.1-NLS-eGFP-p65-flag (regarded as the p65 vector), and pcDNA3.1-azp*-p65- flag or pcDNA3.1-NLSazp*-p65-flag (ATF vectors constructed by our group for another gene [21], regarded as the control ATF vectors) were used as control vectors according to experimental needs.

2.5. Immunofluorescence. The ECV304 cells transfected with ATF vectors were fixed with $4 \%$ paraformaldehyde in phosphate-buffered saline (PBS) and then permeabilized with $0.5 \%$ Triton X-100 in PBS for 10 minutes. After rinsing 3 times with $\mathrm{PBS}$, the cells were incubated in goat serum (1:50 in PBS) for 20 minutes. This was followed by incubation of mouse antiflag monoclonal antibody ( $1: 1000$ in PBS) for 12 hours. The resulting cells were rinsed 3 times with PBS again and finally incubated with goat antimouse IgG, Cy3 conjugate (1:200 in PBS) for 30 minutes. The antibodies were purchased from Boster (Wuhan, China). The resulting cells were then stained with 4,6-diamidino2- phenylindole dihydrochloride (DAPI) (Sigma) solution $(100 \mathrm{ng} / \mathrm{ml}$ in PBS) and finally mounted in $70 \%$ glycerol in PBS containing 2.5\% 1,4-diazabicyclo [2.2.2] octane 


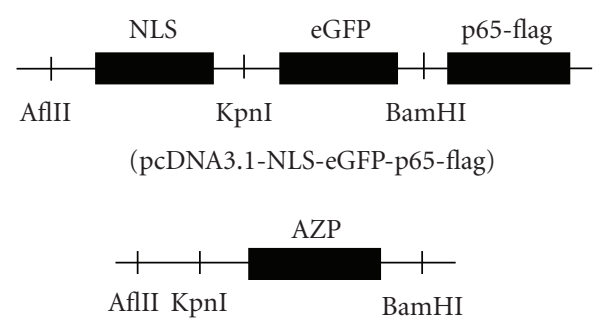

(PUC57-AZP)

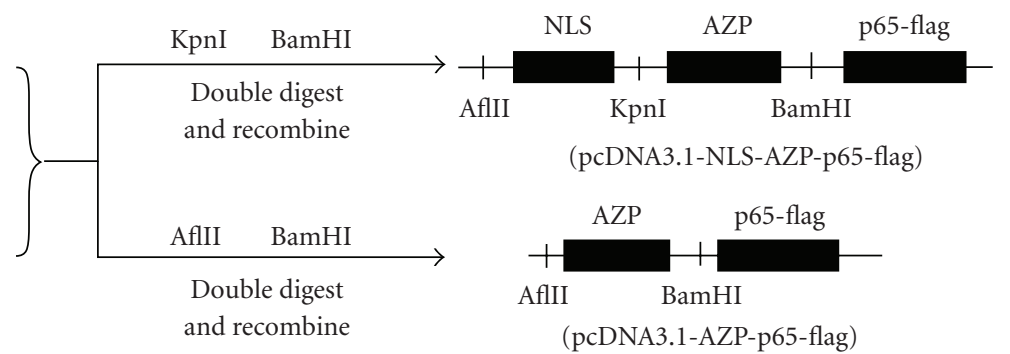

FIgURe 2: Construction of the ATF vectors. PUC57-AZP and pcDNA3.1-NLS-eGFP-p65-flag were double digested at the KpnI (AflII) and BamHI restriction sites respectively to obtain the AZP and pcDNA3.1-p65-flag (pcDNA3.1-NLS-p65-flag) fragments. The two fragments were then recombined to construct pcDNA3.1-NLS-AZP-p65-flag (pcDNA3.1-AZP-p65-flag) vector.

(DABCO) (Sigma). Fluorescence was analyzed under a Leica Tcs Sp5 laser scanning confocal microscope.

2.6. Dual-Luciferase Reporter Assay. The firefly-luciferase reporters were hHO4.9luc and pGL3-control, while the renilla-luciferase reporter was pRL-TK. The hHO4.9luc vector, which was constructed by cloning a $4.9 \mathrm{kbp}$ SacIXhol fragment (containing the promoter and enhancer of human HMOX1) into the pGL3 basic vector, was a kind gift from Professor Norbert Leitinger (University of Virginia, VA, USA) [22]. The pGL3-control vector contains SV40 promoter and enhancer sequences, resulting in strong expression of firefly luciferase in many types of mammalian cells (including ECV304 cells). The candidate vectors were pcDNA3.1-AZP-p65-flag, pcDNA3.1- NLSAZP-p65-flag, PUC57-AZP, pcDNA3.1-NLS-eGFP-p65-flag, and pcDNA3.1-azp* ${ }^{*}$-p65-flag. For each of the vectors, four dose gradients $(0,50 \mathrm{ng}, 100 \mathrm{ng}$, and $150 \mathrm{ng})$ were used. PcDNA3.1 was added to each gradient to reach $200 \mathrm{ng}$ so as to normalize the transfection efficiency. Tritransfection of the candidate vector, firefly-luciferase reporter, and renilla- luciferase reporter were performed on ECV304 cells, which were cultured in 96-well plates. Forty-eight hours after transfection, the dual-luciferase reporter assay was performed using a Dual-Lucy Assay Kit (Vigorous, Beijing, China), according to the protocol provided by the manufacturer. Luminescence intensity was measured by a TD 20/20 Luminometer (Promega, Madison, WI, USA).

2.7. Quantitative Real-Time RT-PCR. Transfections were performed on ECV304 cells, which were cultured in 12 -well plates. The candidate vectors were pcDNA3.1AZP-p65-flag, pcDNA3.1-NLS-AZP- p65-flag, PUC57-AZP, pcDNA3.1-NLS-eGFP-p65-flag, and pcDNA3.1-NLS-azp*p65-flag. For each of the vectors, four dose gradients $(0.5 \mu \mathrm{g}$, $1.0 \mu \mathrm{g}, 1.5 \mu \mathrm{g}$, and $2.0 \mu \mathrm{g}$ ) were used. PcDNA3.1 was added to each gradient to reach $2.0 \mu \mathrm{g}$ so as to normalize the transfection efficiency. Forty-eight hours after transfection, the total RNA was isolated using Trizol reagent (Invitrogen). Total RNA $(0.5 \mu \mathrm{g})$ was used for reverse transcription at $37^{\circ} \mathrm{C}$ for 15 minutes and $85^{\circ} \mathrm{C}$ for 5 seconds. After denaturing at $95^{\circ} \mathrm{C}$ for 10 seconds, PCR was conducted for 40 cycles at $95^{\circ} \mathrm{C}$ for 5 seconds, $55^{\circ} \mathrm{C}$ for 15 seconds, and $60^{\circ} \mathrm{C}$ for 20 seconds. Real-time PCR was performed using a
SYBR PrimeScript RT-PCR Kit (Takara, Shiga, Japan) on a Rotor-Gene 6000 (Gene Company Limited, Hong Kong), according to the protocol provided by the manufacturers. The primers were designed by Primer Premier 5.0 (forward: 5'-ATGACACCAAGGACCAGAGCC-3'; reverse: 5'-GTAAG GACCCATCGGAGAAGC- $3^{\prime}$ ) and were then synthesized at Shanghai Sangon. The amplified product was a $151 \mathrm{bp}$ fragment of HMOX1 cDNA. Human GAPDH was used as the internal control to normalize the results. The GAPDH primers were purchased from Takara (Takara Code D3790, Primer_set_ID HA067812, GenBank Acc. NM_002046). The data were analyzed by Rotor-Gene analysis software.

\section{Results and Discussion}

3.1. Design of the HMOX1 Enhancer-Specific AZP. We used the 54 bp DNA sequence between the two StREs of human HMOX1 enhancer for prescreening (Figure 1). After submitting it to ZF tools $3.0,12$ possible target sites were obtained (Table 1). The sequence (4) 5'-ACT CGC GGA AAC AAA GGG-3', with the second highest score while without TSO, was selected as the best target site for the AZP.

The accessibility of genomic DNA in chromatin to ATFs is very important [23]. Targeting a genomic region around known cis-elements that are accessible by native transcription factors is a good choice when information on the chromatin structures of target genes is not available [24]. Extensive analysis of the mouse gene, and to a lesser extent the human gene, has identified a common mechanism (the StRE/Nrf2 transcription factor pathway) for gene regulation in response to a diverse array of HO-1 inducers [25]. In addition, the StREs were excluded for relative specificity, since the StRE/Nrf2 transcription factor pathway is also involved in the regulation of some other stress-related genes.

The sequence (4) 5'-ACT CGC GGA AAC AAA GGG$3^{\prime}$ was submitted to ZF tools 3.0 again to obtain the amino acid sequence of the corresponding AZP (Figure 3). As a result of "Sequence Feature Scan", "InterproScan" [26] showed that the AZP consisted of six $\mathrm{Cys}_{2}-\mathrm{His}_{2}$ zinc finger motifs, which were located at the 8-30, 36-58, 64-86, 92$114,120-142$, and 148-170 amino acid residues of the AZP. "Secondary Structure \& Disorder prediction" showed the assignment of domains in the AZP and indicated that there were few disordered regions in the AZP. Homology modeling 
TABLe 1: Details of the 12 possible target sites for the AZP.

\begin{tabular}{|c|c|c|}
\hline Target & Score (Base function) & TSO Issues \\
\hline (1) 5'-GGA GGG GGG ACT CGC GGA-3' & 52.95 & Yes \\
\hline (2) 5'-GGG GGG ACT CGC GGA AAC-3' & 56.51 & Yes \\
\hline (3) 5'-GGG ACT CGC GGA AAC AAA-3' & 56.18 & Yes \\
\hline (4) $5^{\prime}$-ACT CGC GGA AAC AAA GGG-3' & 56.18 & No \\
\hline (5) 5'-CGC GGA AAC AAA GGG AAG-3' & 54.35 & Yes \\
\hline (6) 5'-GGA AAC AAA GGG AAG GCG-3' & 52.08 & Yes \\
\hline (7) 5'-AAC AAA GGG AAG GCG GAT-3' & 55.03 & Yes \\
\hline (8) 5'-GCG GAA ACA AAG GGA AGG-3' & 52.26 & No \\
\hline (9) 5'-GAA ACA AAG GGA AGG CGG-3' & 54.03 & No \\
\hline (10) 5'-ACA AAG GGA AGG CGG ATT-3' & 54.43 & No \\
\hline (11) 5'-CGG AAA CAA AGG GAA GGC-3' & 54.19 & No \\
\hline (12) 5'-AAA CAA AGG GAA GGC GGA-3' & 51.03 & No \\
\hline
\end{tabular}

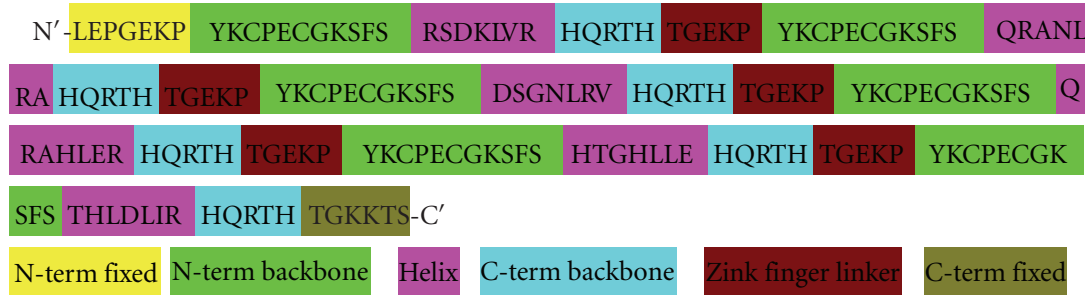

FIgUre 3: The 176 amino acid sequence of the AZP. Different Motifs were shown by distinct background colors.

showed the conformation of the six zinc finger motifs. The modeling was based on the template 2i13A (a stable AZP). The sequence identity was $79.221 \%$, and the $E$-value was $1.26 \mathrm{e}-44$. To each amino acid residue of the AZP, the Anolea value was negative and the Verify $3 \mathrm{D}$ value ranged from 0 to +1 . Taken together, these results suggested that the AZP was quite stable and justified further experiments.

3.2. Construction of the ATF Vectors. The flag tag on the ATF vectors was used for protein expression detection. Immunofluorescence showed that the ECV304 cells, which were transfected with ATF vectors, were Cy3-positive, whereas the control cells were not (Figure 4). This indicated that after vector transfection, the ATF could be normally expressed in ECV304 cells.

An NLS targets the protein to the cell nucleus through the nuclear pore complex and directs a newly synthesized protein into the nucleus via its recognition by cytosolic nuclear transport receptors. The expressed pcDNA3.1-NLSAZP-p65-flag (ATF vector with NLS) was mainly located in the nuclei, while the expressed pcDNA3.1-AZP-p65flag (ATF vector without NLS) was mainly located in the cytoplasm. However, the latter could still be found within the nuclei. These findings are further discussed below.

3.3. Affinity of the ATF to HMOX1 Enhancer. In order to assess the affinity of the ATF to HMOX1 enhancer, a dualluciferase reporter assay was performed. HHO4.9luc and
pGL3-control were used as the firefly-luciferase reporter. In the hHO4.9luc group, for the ATF vectors, a larger dose resulted in a higher luminescence intensity ratio (LIR). At $50 \mathrm{ng}, 100 \mathrm{ng}$, and $150 \mathrm{ng}$ vector dose, the LIR of pcDNA3.1AZP-p65-flag was 1.88-, 2.71-, and 4.00-fold above control, while that of pcDNA3.1-NLS-AZP-p65-flag was 2.04-, 2.67, and 3.88-fold above control (Figure 5(a)). Here, the "fold above control" represented ratios of the LIR of the ECV304 cells transfected with different candidate vectors to that of the ECV304 cells transfected with pcDNA3.1 (control). There were no significant differences between the two ATF vector groups, indicating that the ATF without NLS could also enter the nuclei (which was consistent with the immunofluorescence results) and upregulate the reporter gene expression. This may be attributed to the $\mathrm{N}^{\prime}$-terminal of the p65 functional domain, which acts as an NLS in NF$\kappa \mathrm{B}$. For the three control vectors, LIR showed no significant difference among each dose gradient. In the pGL3-control group, LIR showed no significant difference among each dose gradient for the five candidate vectors (Figure 5(b)).

It is well known that the DBD recognizes a particular region of the DNA, while the ED recruits other proteins of the transcription machinery in the immediate proximity of the DNA to start transcription [10]. Sometimes the DBD may repress gene expression by blocking RNA polymerase movement. However, neither of the two domains alone regulates gene expression effectively. The results of the dualluciferase reporter assay were consistent with the expectations. It was the HMOX1 enhancer-specific ATF, rather than 


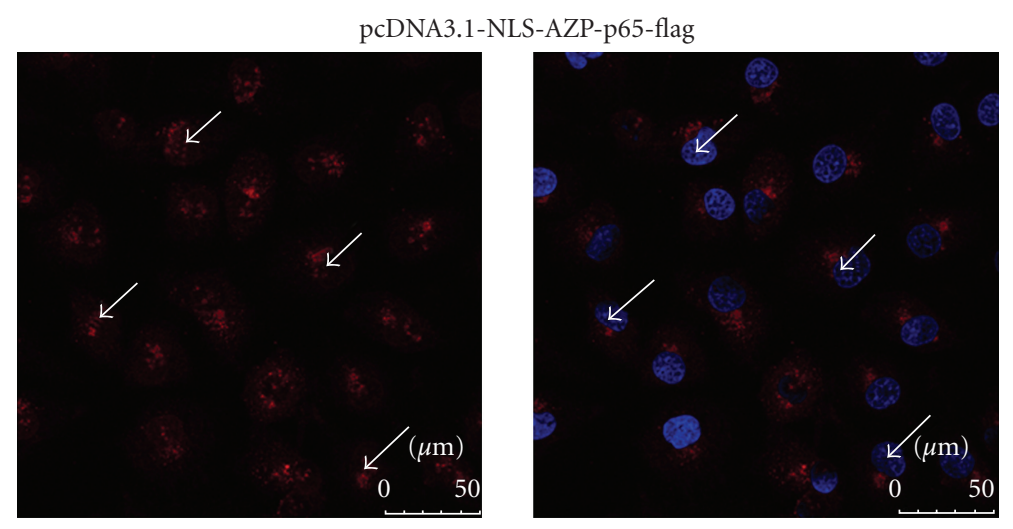

(a)

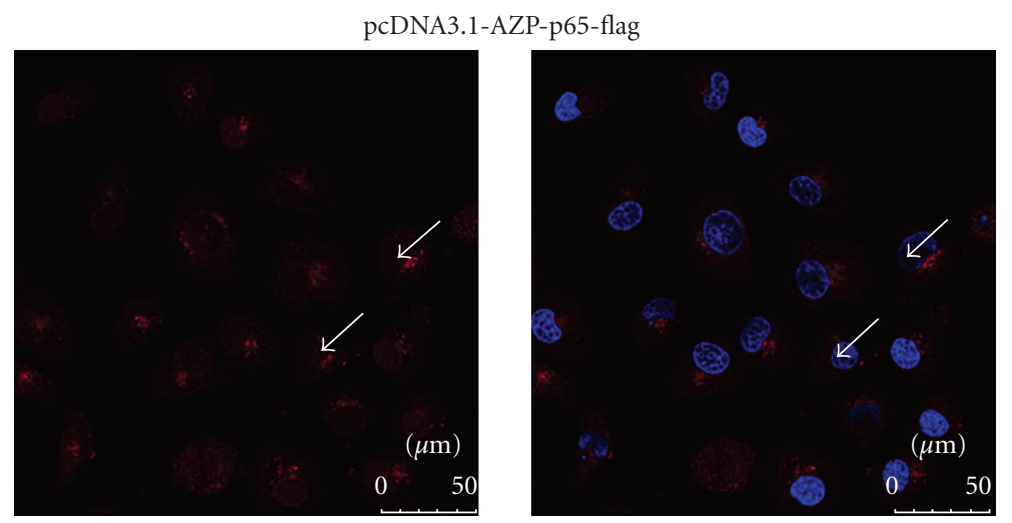

(b)

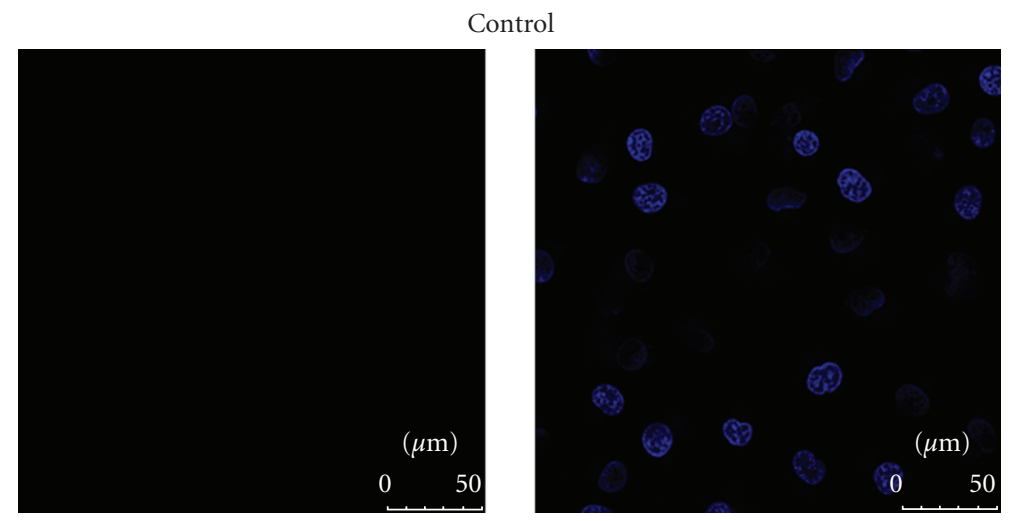

(c)

FIGURE 4: Expression of the ATF in the ECV304 cells. Immunofluorescence was performed, and the resulting cells were observed under a laser scanning confocal microscope. (a) ECV304 cells transfected with pcDNA3.1-NLS-AZP-p65-flag. (b) ECV304 cells transfected with pcDNA3.1-AZP-p65-flag. (c) ECV304 cells transfected with the control plasmids. The white arrows pointed the expressed proteins which were located in the nuclei.

the AZP or the $\mathrm{p} 65$ functional domain alone, that recognized the HMOX1 enhancer and upregulated the reporter gene expression.

3.4. Effect of the ATF on Endogenous HMOX1 Expression. It was reported that ATF which exhibited good performance in transient reporter assays did not effectively modulate the endogenous gene expression [23]. This indicated that the chromatin structures of promoters in reporter plasmids were different from those in actual chromosomes. In order to assess the effect of the ATF on endogenous HMOX1 expression, quantitative real-time RT-PCR was performed. The control vectors were pcDNA3.1-NLS-eGFPp65-flag, PUC57-AZP, and pcDNA3.1-NLS-azp*-p65-flag. Experiments were still done on ECV304 cells rather than 


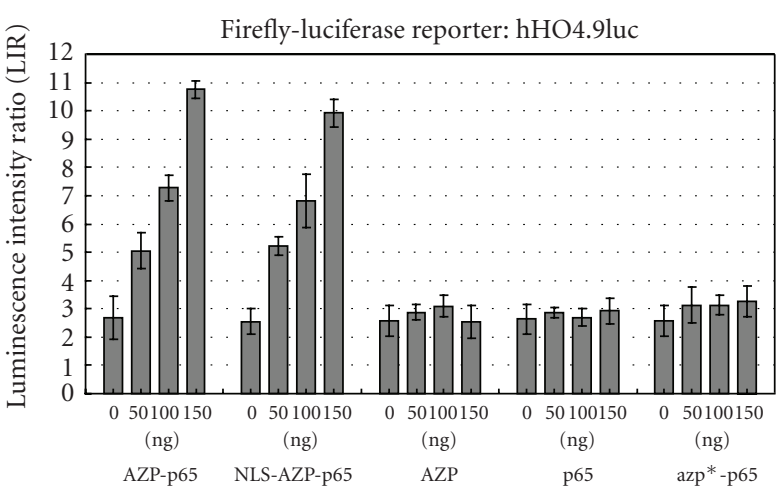

(a)

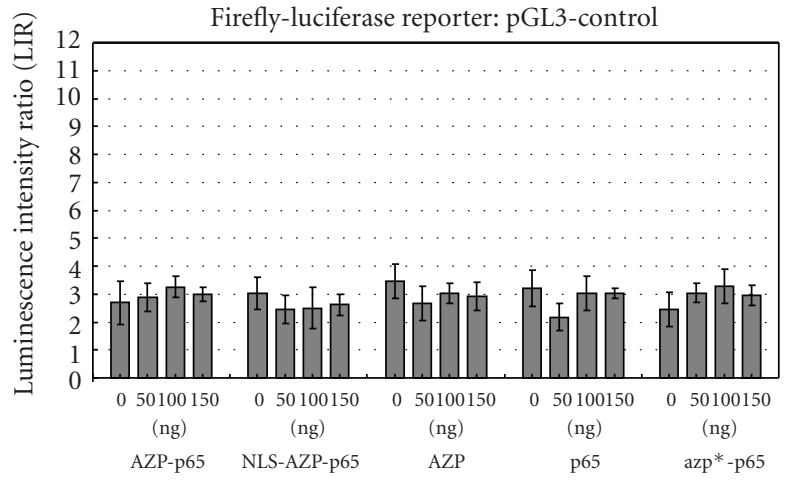

(b)

Figure 5: Affinity of the ATF to HMOX1 enhancer. Dual-luciferase reporter assay was performed; the candidate vectors were pcDNA3.1AZP-p65-flag (AZP-p65), pcDNA3.1-NLS-AZP-p65-flag (NLS-AZP-p65), PUC57-AZP (AZP), pcDNA3.1-NLS-eGFP-p65-flag (p65), and pcDNA3.1- azp*-p65-flag (azp*-p65). For each of the vectors, four dose gradients (0, $50 \mathrm{ng}, 100 \mathrm{ng}$, and $150 \mathrm{ng})$ were used. The fireflyluciferase reporters were (a) hHO4.9luc and (b) pGL3-control, respectively. The data represent the means \pm S.D.

endothelial cell lines (such as HUVECs). This was because HMOX1 was basically expressed in most human cell lines (including ECV304 cells), and ECV304 cells were easy to culture. In the current study, it was a convincing cell line for testing the effect of the ATF on endogenous HMOX1 expression.

For ATF vectors, HMOX1 mRNA levels increased with the vector dose, and a dose-response relationship existed. At $0.5 \mu \mathrm{g}, 1.0 \mu \mathrm{g}, 1.5 \mu \mathrm{g}$, and $2.0 \mu \mathrm{g}, \mathrm{HMOX} 1 \mathrm{mRNA}$ of the pcDNA3.1-AZP-p65-flag group was 3.19-, 4.06-, 4.75-, and 5.91-fold above control, while that of the pcDNA3.1-NLSAZP-p65-flag group was 4.71-, 5.90-, 9.12-, and 10.28-fold above control (Figure 6). Here, the "fold above control" represented ratios of the HMOX1 mRNA level of the ECV304 cells transfected with different candidate vectors to that of the ECV304 cells transfected with pcDNA3.1 (control). Different from the dual-luciferase reporter assay results, the ATF with NLS seemed to be more efficient than that without NLS in upregulating endogenous HMOX1 expression. This may be attributed to the differences in the nature of the two assays. In contrast, the three control vectors failed to raise HMOX-1 mRNA levels at any dose gradient. These indicated that the HMOX1 enhancer-specific ATF could upregulate endogenous HMOX1 expression, as expected. The ATF with NLS was more efficient than that without NLS.

In the current study, the p65 functional domain was used as the ED. In addition, other activation domains, such as VP16, VP64, p65, S3H, and (FDTDL) 11 , may also be considered and may result in a more potent ATF. Moreover, repressor domains, such as KRAB, SID, ERD, vErbA, and SRDX fused to the HMOX1 enhancer-specific AZP, should prevent HMOX1 from activation [27]. However, these repressor domains should be verified by further experiments.

Most human diseases can be treated by activating or repressing endogenous gene expression. Thus, ATF technology has extensive therapeutic applications. It differs from many novel methods, such as RNA interference or antisense

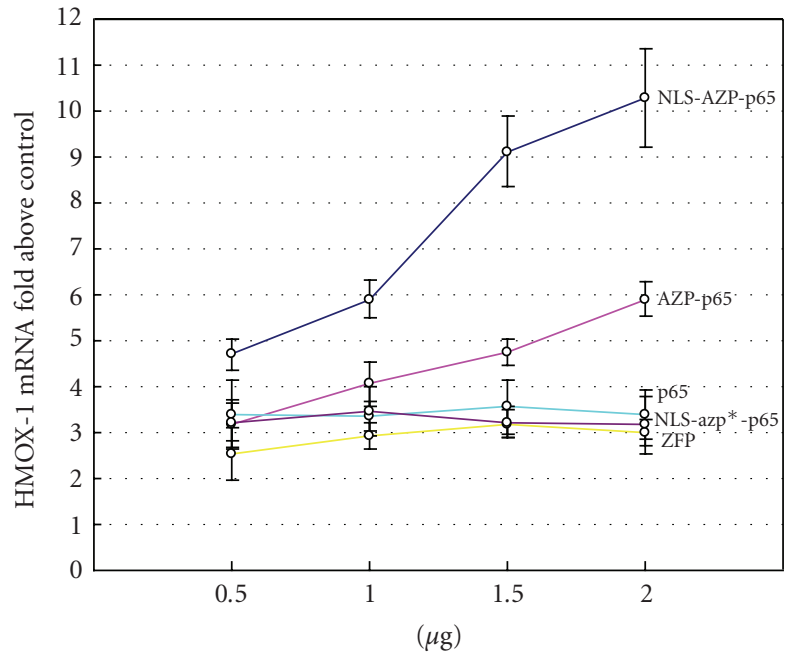

Figure 6: Effect of the ATF on endogenous HMOX1 expression. Quantitative real-time RT-PCR was performed; the candidate vectors were pcDNA3.1-AZP-p65-flag (AZP-p65), pcDNA3.1-NLSAZP-p65-flag (NLS-AZP-p65), PUC57-AZP (AZP), pcDNA3.1NLS-eGFP-p65-flag (p65), and pcDNA3.1-NLS-azp*-p65-flag (NLS-azp*-p65). For each of them, four dose gradients $(0.5 \mu \mathrm{g}$, $1.0 \mu \mathrm{g}, 1.5 \mu \mathrm{g}$, and $2.0 \mu \mathrm{g}$ ) were used. The data represent the means \pm S.D.

technology, for it can be used for the gene expression upregulation rather than exclusively for its downregulation [28]. Our work has shown that an HMOX1 enhancerspecific AZP could be readily designed by bioinformatical methods. By fusing the AZP to the p65 functional domain with an NLS, the resulting ATF could upregulate endogenous HMOX1 expression in ECV304 cells. This approach provides a facile way to modulate endogenous gene expression. It can be applied to other disease-related genes as well. We believe that with further research aimed at selecting the most potent activation domain and acquiring the cell-penetrating 
protein, the ATF could be developed as a potential drug for cardiovascular diseases.

\section{Acknowledgments}

This work was supported by the Natural Science Foundation in China (no. 30672535). The authors would also like to express their sincere appreciation to Dr. Philip Gregory for providing the eukaryotic expression vector, pcDNA3.1-NLSeGFP-p65- flag, and Professor Norbert Leitinger for providing the firefly-luciferase reporter gene vector, hHO4.9luc.

\section{References}

[1] D. Morse and A. M. K. Choi, "Heme oxygenase-1: the "emerging molecule" has arrived," American Journal of Respiratory Cell and Molecular Biology, vol. 27, no. 1, pp. 8-16, 2002.

[2] J. F. Ndisang, L. Wu, W. Zhao, and R. Wang, "Induction of heme oxygenase-1 and stimulation of cGMP production by hemin in aortic tissues from hypertensive rats," Blood, vol. 101, no. 10, pp. 3893-3900, 2003.

[3] C.-M. Hu, Y.-H. Chen, M.-T. Chiang, and L.-Y. Chau, "Heme oxygenase-1 inhibits angiotensin II-induced cardiac hypertrophy in vitro and in vivo," Circulation, vol. 110, no. 3, pp. 309-316, 2004.

[4] K. Ishikawa, D. Sugawara, X.-P. Wang et al., "Heme oxygenase1 inhibits atherosclerotic lesion formation in LDL-receptor knockout mice," Circulation Research, vol. 88, no. 5, pp. 506$512,2001$.

[5] S.-H. Juan, T.-S. Lee, K.-W. Tseng et al., "Adenovirus-mediated heme oxygenase-1 gene transfer inhibits the development of atherosclerosis in apolipoprotein e-deficient mice," Circulation, vol. 104, no. 13, pp. 1519-1525, 2001.

[6] A. Piperno, P. Trombini, M. Gelosa et al., "Increased serum ferritin is common in men with essential hypertension," Journal of Hypertension, vol. 20, no. 8, pp. 1513-1518, 2002.

[7] N. Lindenblatt, R. Bordel, W. Schareck, M. D. Menger, and B. Vollmar, "Vascular heme oxygenase-1 induction suppresses microvascular thrombus formation in vivo," Arteriosclerosis, Thrombosis, and Vascular Biology, vol. 24, no. 3, pp. 601-606, 2004.

[8] S. R. Vulapalli, Z. Chen, B. H. L. Chua, T. Wang, and C.-S. Liang, "Cardioselective overexpression of $\mathrm{HO}-1$ prevents I/Rinduced cardiac dysfunction and apoptosis," American Journal of Physiology, vol. 283, no. 2, pp. H688-H694, 2002.

[9] R. R. Beerli and C. F. Barbas III, "Engineering polydactyl zincfinger transcription factors," Nature Biotechnology, vol. 20, no. 2, pp. 135-141, 2002.

[10] Z. Wu, G. Belanger, B. B. Brennan et al., "Targeting the transcriptional machinery with unique artificial transcriptional activators," Journal of the American Chemical Society, vol. 125, no. 41, pp. 12390-12391, 2003.

[11] T. Sera, "Zinc-finger-based artificial transcription factors and their applications," Advanced Drug Delivery Reviews, vol. 61, no. 7-8, pp. 513-526, 2009.

[12] M. Isalan and Y. Choo, "Rapid, high-throughput engineering of sequence-specific zinc finger DNA-binding proteins," Methods in Enzymology, vol. 340, pp. 593-609, 2001.

[13] T. Sera and C. Uranga, "Rational design of artificial zincfinger proteins using a nondegenerate recognition code table," Biochemistry, vol. 41, no. 22, pp. 7074-7081, 2002.
[14] J. G. Mandell and C. F. Barbas III, "Zinc finger tools: custom DNA-binding domains for transcription factors and nucleases," Nucleic Acids Research, vol. 34, pp. W516-W523, 2006.

[15] K. Arnold, L. Bordoli, J. Kopp, and T. Schwede, "The SWISSMODEL workspace: a web-based environment for protein structure homology modelling," Bioinformatics, vol. 22, no. 2, pp. 195-201, 2006.

[16] T. Schwede, J. Kopp, N. Guex, and M. C. Peitsch, "SWISSMODEL: an automated protein homology-modeling server," Nucleic Acids Research, vol. 31, no. 13, pp. 3381-3385, 2003.

[17] F. Kiefer, K. Arnold, M. Künzli, L. Bordoli, and T. Schwede, "The SWISS-MODEL repository and associated resources," Nucleic Acids Research, vol. 37, no. 1, pp. D387-D392, 2009.

[18] F. Melo and E. Feytmans, "Assessing protein structures with a non-local atomic interaction energy," Journal of Molecular Biology, vol. 277, no. 5, pp. 1141-1152, 1998.

[19] D. Eisenberg, R. Lüthy, and J. U. Bowie, "VERIFY3D: assessment of protein models with three-dimensional profiles," Methods in Enzymology, vol. 277, pp. 396-406, 1997.

[20] H. G. Drexler, H. Quentmeier, W. G. Dirks et al., "Bladder carcinoma cell line ECV304 is not a model system for endothelial cells," In Vitro Cellular and Developmental Biology, vol. 38, no. 4, pp. 185-187, 2002.

[21] Y. Wei, D. Ying, C. Hou, X. Cui, and C. Zhu, "Design of a zinc finger protein binding a sequence upstream of the A20 gene," BMC Biotechnology, vol. 8, article no. 28, 2008.

[22] G. Krönke, V. N. Bochkov, J. Huber et al., "Oxidized phospholipids induce expression of human heme oxygenase1 involving activation of cAMP-responsive element-binding protein," Journal of Biological Chemistry, vol. 278, no. 51, pp. 51006-51014, 2003.

[23] P.-Q. Liu, E. J. Rebar, L. Zhang et al., "Regulation of an endogenous locus using a panel of designed zinc finger proteins targeted to accessible chromatin regions: activation of vascular endothelial growth factor A," Journal of Biological Chemistry, vol. 276, no. 14, pp. 11323-11334, 2001.

[24] T. Gräslund, X. Li, L. Magnenat, M. Popkov, and C. F. Barbas III, "Exploring strategies for the design of artificial transcription factors," Journal of Biological Chemistry, vol. 280, no. 5, pp. 3707-3714, 2005.

[25] J. Alam and J. L. Cook, "Transcriptional regulation of the heme oxygenase-1 gene via the stress response element pathway," Current Pharmaceutical Design, vol. 9, no. 30, pp. 2499-2511, 2003.

[26] E. M. Zdobnov and R. Apweiler, "InterProScan-an integration platform for the signature-recognition methods in InterPro," Bioinformatics, vol. 17, no. 9, pp. 847-848, 2001.

[27] K. Tachikawa, O. Schröder, G. Frey, S. P. Briggs, and T. Sera, "Regulation of the endogenous VEGF-A gene by exogenous designed regulatory proteins," Proceedings of the National Academy of Sciences of the United States of America, vol. 101, no. 42, pp. 15225-15230, 2004.

[28] A. Z. Ansari, "Fingers reach for the genome," Nature Biotechnology, vol. 21, no. 3, pp. 242-243, 2003. 

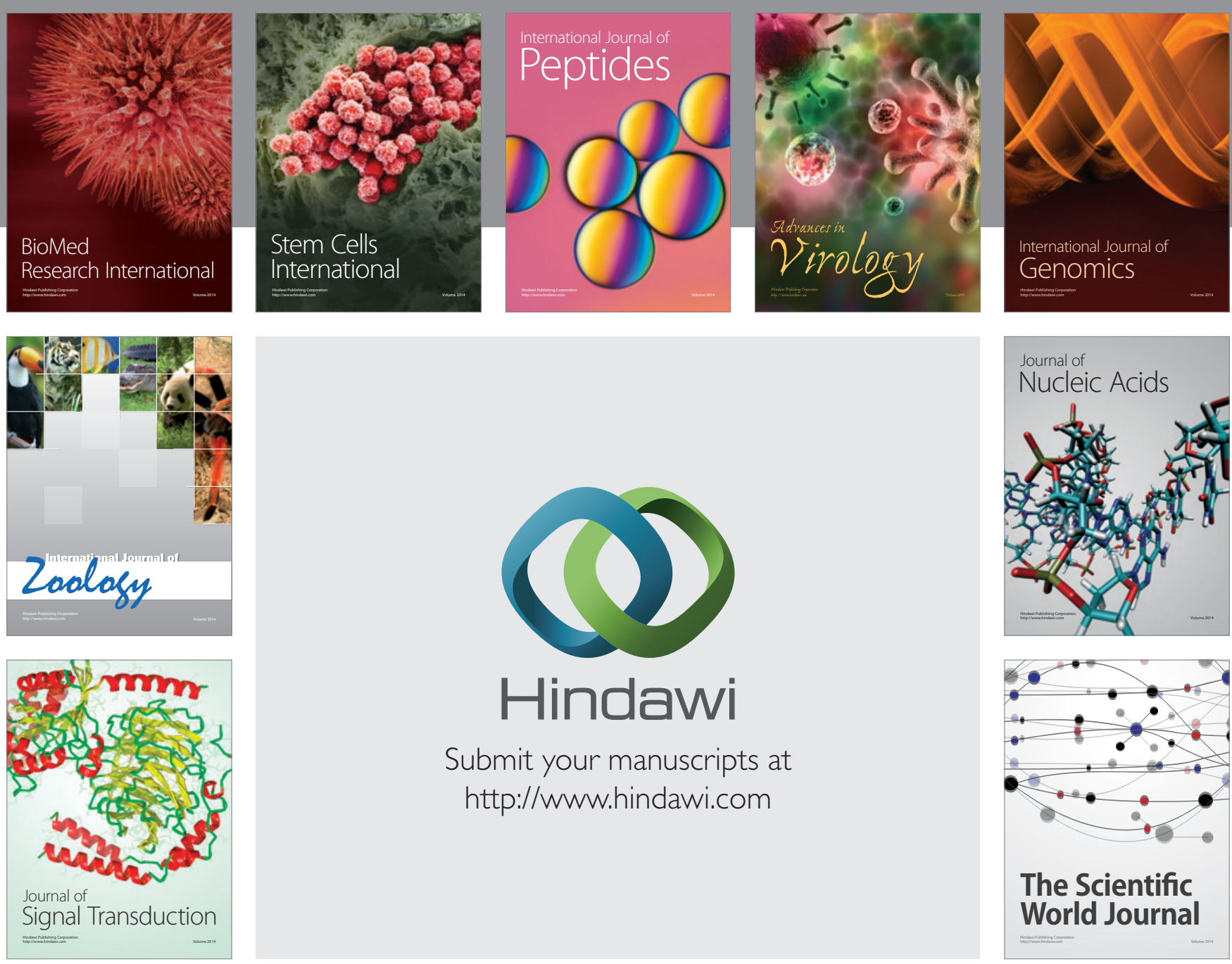

Submit your manuscripts at

http://www.hindawi.com
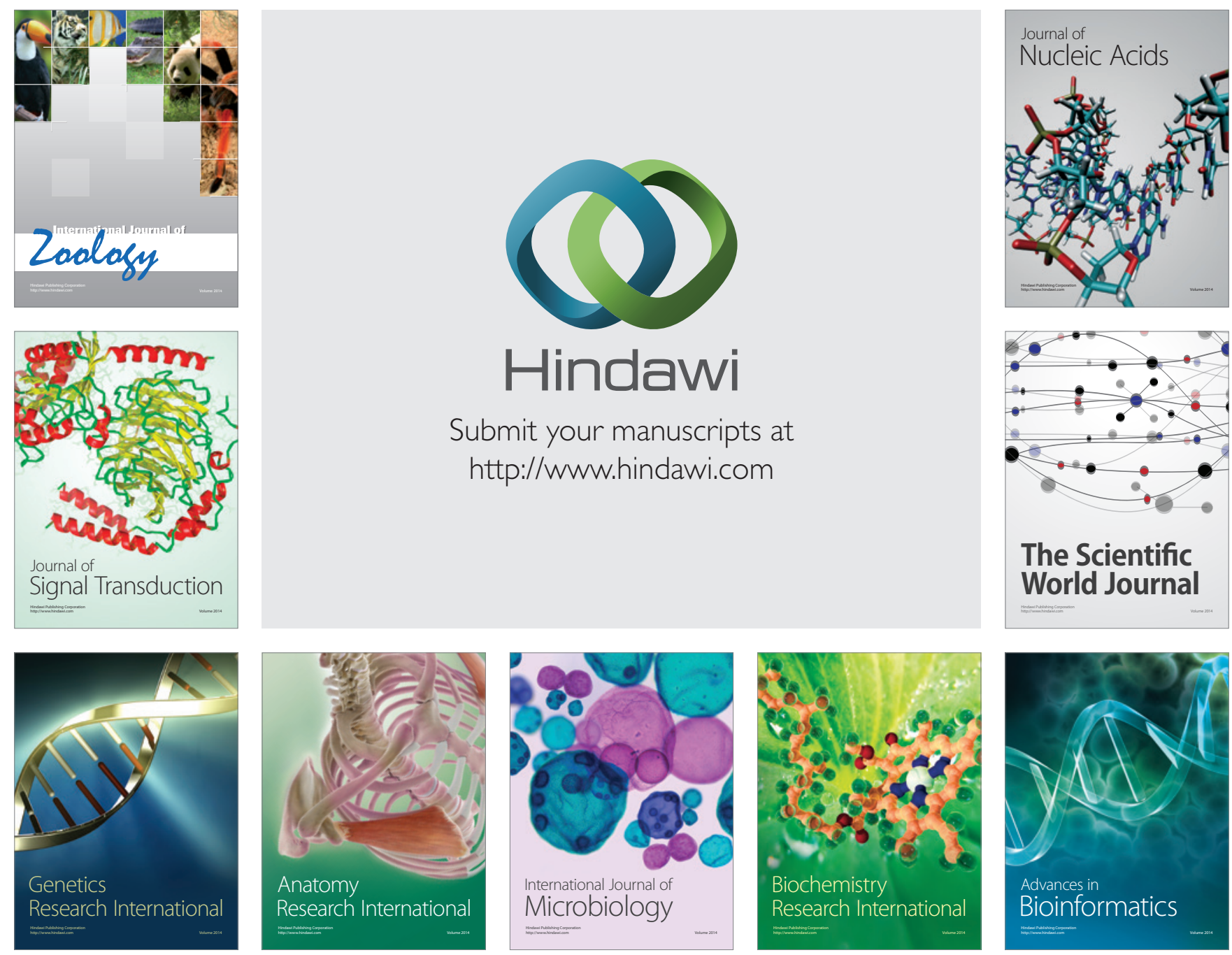

The Scientific World Journal
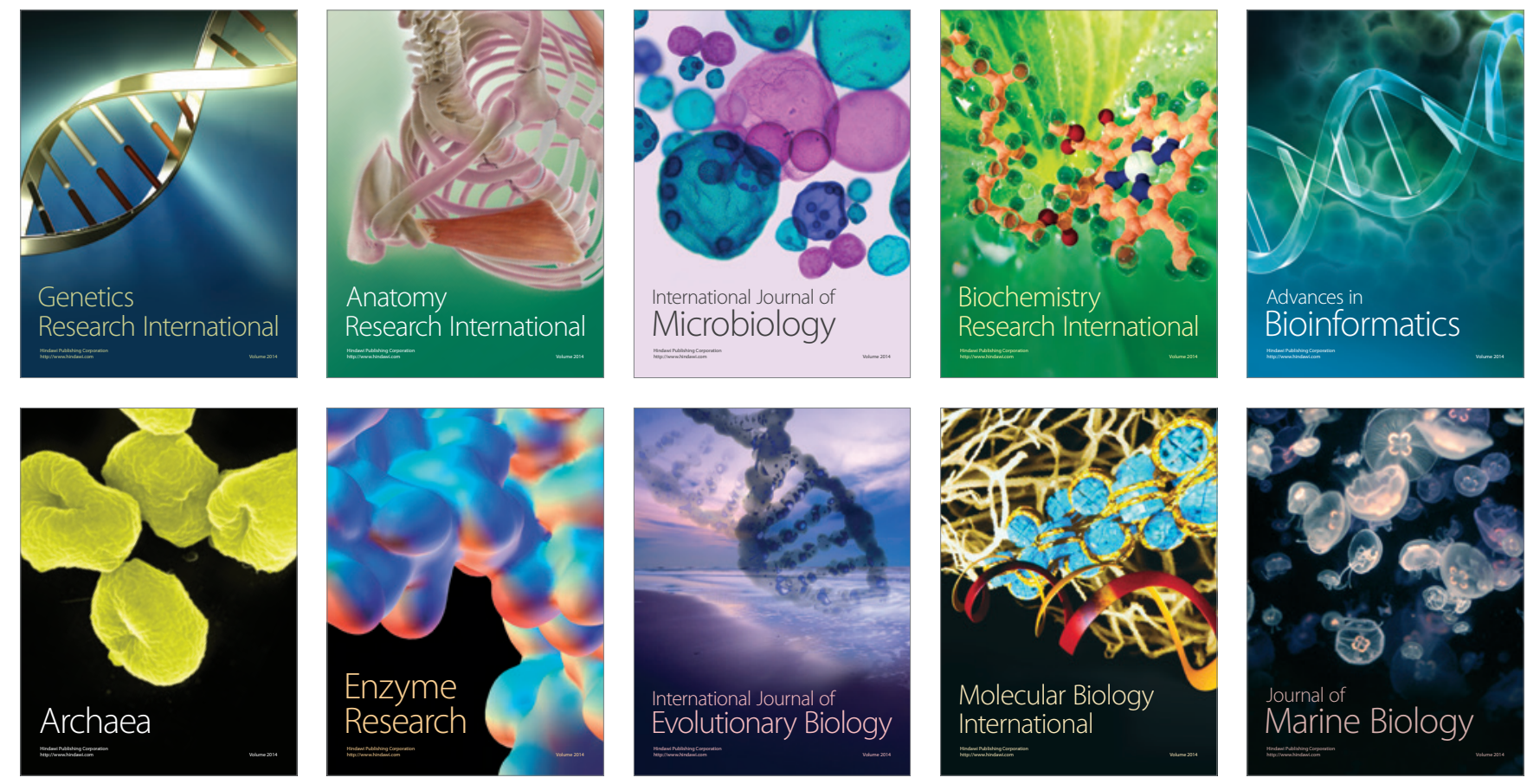